\title{
Comparison of the Lysis Centrifugation Method with the Conventional Blood Culture Method in Cases of Sepsis in a Tertiary Care Hospital
}

\author{
Harshal R Parikh, Anuradha S De, Sujata M Baveja
}

Department of Microbiology, L. T. M. Medical College, Sion, Mumbai, Maharashtra, India

Address for correspondence: Dr. Anuradha De, E-mail: dr_anuradhade@yahoo.com

\section{ABSTRACT}

Introduction: Physicians and microbiologists have long recognized that the presence of living microorganisms in the blood of a patient carries with it considerable morbidity and mortality. Hence, blood cultures have become critically important and frequently performed test in clinical microbiology laboratories for diagnosis of sepsis.

Objectives: To compare the conventional blood culture method with the lysis centrifugation method in cases of sepsis. Materials and Methods: Two hundred nonduplicate blood cultures from cases of sepsis were analyzed using two blood culture methods concurrently for recovery of bacteria from patients diagnosed clinically with sepsis - the conventional blood culture method using trypticase soy broth and the lysis centrifugation method using saponin by centrifuging at $3000 \mathrm{~g}$ for 30 minutes.

Results: Overall bacteria recovered from 200 blood cultures were $17.5 \%$. The conventional blood culture method had a higher yield of organisms, especially Gram positive cocci. The lysis centrifugation method was comparable with the former method with respect to Gram negative bacilli. The sensitivity of lysis centrifugation method in comparison to conventional blood culture method was $49.75 \%$ in this study, specificity was $98.21 \%$ and diagnostic accuracy was $89.5 \%$. In almost every instance, the time required for detection of the growth was earlier by lysis centrifugation method, which was statistically significant. Contamination by lysis centrifugation was minimal, while that by conventional method was high. Time to growth by the lysis centrifugation method was highly significant $(P$ value 0.000$)$ as compared to time to growth by the conventional blood culture method.

Conclusion: For the diagnosis of sepsis, combination of the lysis centrifugation method and the conventional blood culture method with trypticase soy broth or biphasic media is advocable, in order to achieve faster recovery and a better yield of microorganisms.

Key words: Blood culture, lysis centrifugation, saponin

$\varnothing$ nvasion of the bloodstream by living microorganism causes significant morbidity and mortality, a fact well recognized before the dawn of modern medicine. Hence, blood cultures have become and still remain an essential requisite for diagnosis of sepsis. ${ }^{[1]}$ A positive blood culture not only suggests a definitive diagnosis but also guides specific therapy and prognosis. ${ }^{[2]}$ The final identification of a microorganism by the conventional blood culture

\begin{tabular}{|l|l|}
\hline \multicolumn{2}{c}{ Access this article online } \\
\hline Quick Response Code: & Website: \\
\hline & www.jponline.org \\
\hline & \\
\hline
\end{tabular}

method takes about 2 to 3 days, provided the organism grows in the first subculture done within 6 to $18 \mathrm{~h}$ after the receipt of the sample. ${ }^{[3]}$ The lysis centrifugation method concentrates and separates microorganism from plasma, thereby getting separated from antibiotics and other antibacterial factors that may also be present in blood. The presence of actual colonies after initial incubation for direct identification, not only makes it rapid but one can also quantify the colony forming units. Based on clinical impression, special media can be chosen for the initial culture setup. It has been found to be an excellent method for intracellular organisms including yeasts and thermal dimorphic fungi. Processing specimens in biological safety cabinets significantly reduces contamination rates, a drawback well known of this method apart 
from being labor intensive. It is not an optimal method for recovering anaerobes, Haemophilus influenzae, listeria and pneumococci. ${ }^{[4]}$

There are few international studies on the lysis centrifugation method that showed it to be a better method than the conventional one. ${ }^{[5-9]} \mathrm{Few}$ Indian reports using lysis centrifugation method for brucella, ${ }^{[10]}$ mycobacteria ${ }^{[11]}$ and fung ${ }^{[12]}$ are available. There is no documented Indian study of recovery of commonly encountered bacteria in blood cultures by lysis centrifugation method. Hence, this study was undertaken with the intention of comparing the lysis centrifugation method with the conventional blood culture method in terms of yield and recovery rate of bacteria.

\section{MATERIALS AND METHODS}

Two hundred patients admitted with clinical signs and symptoms of sepsis in a tertiary care hospital from January 2009 to June 2010 were studied. Requisite amount of blood was collected aseptically during the fever spike or just before the next dose of an antibiotic, simultaneously for lysis centrifugation and conventional blood culture methods [Table 1]. By the conventional method, blood was inoculated in blood culture bottles containing trypticase soy broth (1:5 dilutions). They were incubated at $35^{\circ} \mathrm{C}$ for 7 days and periodically shaken. The first subculture was done from each bottle within 12-24 h on Blood agar and MacConkey agar plates and then on fourth and seventh day. ${ }^{[3,4]}$ In the lysis centrifugation method, blood was collected in $10 \mathrm{~mL}$ screw capped tubes containing $0.2 / 0.5 \mathrm{~mL}$ solution of saponin $(2 / 5 \mathrm{mg})$ and sodium polyanetholsulfonate (SPS) $(0.8 / 2 \mathrm{mg})$ for pediatric and adult patients, respectively. ${ }^{[13]}$

All the screw capped tubes containing saponin, in which blood was collected were centrifuged within two hours for 30 minutes at $3000 \mathrm{~g}$ ( $\mathrm{g}=$ gravitational force). Supernatant was discarded using sterile Pasteur pipette. Around $0.5 \mathrm{~mL}$ of the sediment was spot inoculated on blood agar and MacConkey agar plates and then with sterile nichrome loop, hexagonal streaking was done. Both the plates were then incubated at $35^{\circ} \mathrm{C}$ aerobically overnight. The plates were examined for any growth. If there was no growth, they were further incubated for $24 \mathrm{~h}$ after which they were discarded. ${ }^{[4]}$

If there was growth by either the conventional or lysis centrifugation method, the organism was further identified by standard laboratory techniques. ${ }^{[13]}$

\section{RESULTS}

From 200 blood cultures included in this study, 17.5\% isolates were recovered both by the lysis centrifugation method and conventional method. The ability of each system to recover the species observed in this study is shown in Table 2. The conventional blood culture method recovered more organisms $(16 \%)$ than the lysis centrifugation method (8.5\%). By Wilson's score, sensitivity of the lysis centrifugation method for Gram-positive cocci was very less $(14.29 \%)$ but specificity was very high $(99.46 \%)$; while sensitivity of lysis centrifugation method for Gram-negative bacilli was $64.71 \%$ but specificity was very high $(99.45 \%)$, as compared to the conventional blood culture method. Except Staphylococcus aureus, other Grampositive cocci (Enterococcus and Streptococcus species) could not be recovered by the lysis centrifugation method.

Time required for the growth of organisms grown by both lysis centrifugation and conventional blood culture method is shown in Table 3. Gram-positive cocci grew within $18 \mathrm{~h}$ approximately by the lysis centrifugation method as compared to $42 \mathrm{~h}$ (approx.) by the conventional method. Gram-negative bacilli grew within 16-24 h by

\begin{tabular}{lccc}
\hline $\begin{array}{l}\text { Table 1: Amount of blood collected by lysis } \\
\text { centrifugation method and conventional blood } \\
\text { culture method } \\
\text { menn }\end{array}$ & Total \\
\cline { 2 - 3 } Age range & \multicolumn{2}{c}{ Amount of blood collected } & \\
\cline { 2 - 3 } & $\begin{array}{c}\text { Conventional } \\
\text { method }\end{array}$ & $\begin{array}{c}\text { Lysis centrifugation } \\
\text { method }\end{array}$ & \\
\hline 0-1 years & $1 \mathrm{~mL}$ & $1 \mathrm{~mL}$ & $2 \mathrm{~mL}$ \\
$1-14$ years & $2 \mathrm{~mL}$ & $2 \mathrm{~mL}$ & $4 \mathrm{~mL}$ \\
$>14$ years & $5 \mathrm{~mL}$ & $5 \mathrm{~mL}$ & $10 \mathrm{~mL}$ \\
\hline
\end{tabular}

\begin{tabular}{lccc}
\hline $\begin{array}{l}\text { Table 2: Number of isolates by lysis } \\
\text { centrifugation and conventional } \\
\text { methods } \\
\text { Organism }\end{array}$ & $\begin{array}{c}\text { By lysis } \\
\text { centrifugation } \\
\text { method }\end{array}$ & $\begin{array}{c}\text { By } \\
\text { conventional } \\
\text { method }\end{array}$ & Both \\
\hline Klebsiella pneumoniae & 00 & 01 & 06 \\
Escherichia coli & 00 & 00 & 02 \\
Enterobacter species & 00 & 00 & 01 \\
Salmonella typhi & 00 & 01 & 00 \\
Pseudomonas aeruginosa & 00 & 01 & 01 \\
Acinetobacter species & 01 & 03 & 01 \\
Methicillin resistant & 00 & 04 & 02 \\
Staphylococcus aureus (MRSA) & 01 & 03 & 00 \\
Methicillin sensitive & 00 & 04 & 00 \\
Staphylococcus aureus (MSSA) & 00 & 04 & 00 \\
Enterococcus species & 00 & 01 & 01 \\
Streptococcus species & 01 & 00 & 14 \\
Candida parapsilosis & 03 & 18 & \\
Total & &
\end{tabular}




\begin{tabular}{|c|c|c|}
\hline \multicolumn{3}{|c|}{$\begin{array}{l}\text { Table } 3 \text { : Time required for growth of } \\
\text { organisms by both lysis centrifugation and } \\
\text { conventional blood culture method }\end{array}$} \\
\hline Organisms grown & $\begin{array}{l}\text { Lysis centrifugation } \\
\text { method (hours) }\end{array}$ & $\begin{array}{l}\text { Conventional } \\
\text { method (hours) }\end{array}$ \\
\hline K. pneumoniae & 18 & 42 \\
\hline K. pneumoniae & 20 & 44 \\
\hline K. pneumoniae & 18 & 42 \\
\hline K. pneumoniae & 16 & 40 \\
\hline K. pneumoniae & 16 & 40 \\
\hline K. pneumoniae & 19 & 43 \\
\hline E. coli & 20 & 44 \\
\hline E. coli & 20 & 44 \\
\hline Enterobacter species & 22 & 46 \\
\hline P. aeruginosa & 21 & 45 \\
\hline Acinetobacter species & 24 & 96 \\
\hline MRSA & 18 & 42 \\
\hline MRSA & 18 & 42 \\
\hline C. parapsilosis & 48 & 96 \\
\hline
\end{tabular}

lysis centrifugation method as compared to $40-96$ h by the conventional method. Candida species required double time in the conventional method ( $96 \mathrm{~h}$ ) as compared to the lysis centrifugation method (48 h). By Chi square analysis (Yates correction), time to growth by lysis centrifugation was highly significant $(P$ value $=0.000)$, as compared to time to growth by the conventional blood culture method.

Total contamination by lysis centrifugation method was low $(0.5 \%)$, as compared to the conventional blood culture method $(7.0 \%)$.

\section{DISCUSSION}

Overall bacteria recovered from 200 cases of sepsis were $17.5 \%$. A study from Nepal has reported the same in $20 \%$, which is almost similar to the present study. ${ }^{[14]}$ Culture positivity by conventional blood culture method was $16 \%$ in this study. A study from Chandigarh has reported overall blood culture positivity of $9.94 \%,{ }^{[15]}$ which is less than this study. Culture positivity by the lysis centrifugation method was $8.5 \%$ in this study. Different studies from USA have shown a better recovery of bacteria by the lysis centrifugation method, in comparison to the conventional blood culture method. ${ }^{[5,7-9]}$ In this study, however, the conventional blood culture method yielded greater culture positivity $(16 \%)$, than the lysis centrifugation method. This might be explained that all studies in USA used the Isolator system (Wampole Laboratories/Dupont) containing inert fluorochemical apart from saponin, whereas in this study only saponin was used. By Wilson's score, the sensitivity of the lysis centrifugation method in comparison to the conventional blood culture method was $49.75 \%$ in this study, specificity was $98.21 \%$ and diagnostic accuracy was $89.5 \%$.
As far as Indian studies are considered, the lysis centrifugation method was compared with The conventional blood culture method for diagnosis of brucellosis and fungal sepsis and both these studies showed a higher positivity by the former method. ${ }^{[10,12]}$ In another Indian study from Vellore, 16.2\% Mycobacterium tuberculosis were detected by the lysis centrifugation method. ${ }^{[1]]}$ Brucella species, $M$. tuberculosis and fungi are all intracellular microorganisms, but in this study only the common bacterial pathogens of sepsis, which are extracellular were looked for. Thus, this study revealed that the lysis centrifugation method is not suitable for extracellular microorganisms.

Kelly et al. have reported 3\% contamination by the lysis centrifugation method. ${ }^{[7]}$ By the conventional method, contamination as reported by Henry et al. was slightly higher $(3.2 \%)$ than the lysis centrifugation method $(3 \%) .{ }^{[9]}$ However, few studies have reported greater contamination rates with the lysis centrifugation method (4.4-9.6\%), than conventional method $(0.6-2.7 \%) .{ }^{[5,7-9]}$ In this study, contamination was remarkably less $(0.5 \%$ only) by the lysis centrifugation method. Contamination in the conventional method could have been reduced, if Castaneda's method of blood culture (biphasic media) was used instead.

According to the standards published by the American Society for Microbiology, the rate of blood culture contamination should not exceed 3\%. ${ }^{[16]}$ Therefore, in this study, the contamination rate by the conventional method is not acceptable. A recent study from the United Kingdom has also reported $7.4 \%$ contamination from blood cultures. ${ }^{[17]}$ Therefore, this highlights the need for regular training and education of health care professionals, who collect blood samples and laboratory technicians who process the samples.

Except few Staphylococcus aureus, other Gram-positive cocci like enterococci and Streptococcus species could not be recovered by lysis centrifugation method.

Though Kelly et al. have reported that both the methods were equally effective for detection of $S$. aureus, the conventional blood culture method was more effective for detection of Streptococcus species. ${ }^{[7]}$ In this study, sensitivity of the lysis centrifugation method for Gram-negative bacilli was higher than that of Gram-positive cocci (64.71\%), as compared to the conventional blood culture method. Henry et al. also reported significant recovery of $S$. aureus by lysis centrifugation method, but Streptococcus species was significantly recovered by conventional blood culture, ${ }^{[9]}$ which is similar to this study. Therefore, the lysis centrifugation method can be used for recovery of 
Gram-negative bacilli and S. aureus and the conventional blood culture method is more effective for the detection of streptococci and enterococci.

Amongst the Gram-negative bacilli, Klebsiella pneumoniae predominated with a recovery rate of $41.18 \%(7 / 17)$ by the conventional blood culture method and 50\% (6/12) by lysis centrifugation method. De et al. have also isolated K. pneumoniae as the commonest Gram-negative bacilli (34.3\%) from blood cultures of hospitalized children suspected of sepsis. ${ }^{[18]}$ Acinetobacter species was recovered more by the conventional blood culture method. Candida species was recovered more by lysis centrifugation method. Sinha et al. have also reported significant recovery of Candida species by the lysis centrifugation method as compared to the conventional method. ${ }^{[12]}$

Bacteria grown by only conventional blood culture method took minimum $48 \mathrm{~h}$ to grow, whereas bacteria by only lysis centrifugation method grew within 18-24 h.

Time to growth by the lysis centrifugation method for all Enterobacteriaceae and S. aureus was 1 day $(24 \mathrm{~h})$ earlier as compared to the conventional blood culture method [Table 3]. By t-test, two-sample assuming equal variance, time to growth for Enterobacteriaceae was significant $(P<0.000)$ by the lysis centrifugation method as compared to the conventional blood culture method. For nonfermenters and Candida species, it was 2 days ( $48 \mathrm{~h}$ ) earlier by the former method [Table 3]. Kiehn et al. ${ }^{[8]}$ have also reported the superiority of the lysis centrifugation method over the conventional blood culture method by detecting the bacteria on an average of 1 day earlier and yeasts on an average of 2 days earlier, which is exactly similar to this study. Henry et al. have detected Grampositive cocci and Pseudomonas aeruginosa 1 day earlier by the lysis centrifugation method. ${ }^{[9]}$ However, in this study, the nonfermenters were detected 2 days earlier by the lysis centrifugation method [Table 2]. Other reports from USA have also highlighted the early recovery of bacteria as well as fungi by the lysis centrifugation method. ${ }^{[-7]}$

In this study, the lysis centrifugation method did not turn out to be a better method than the conventional blood culture method for the detection of bacteria from blood, and for the diagnosis of sepsis. Nevertheless, the time taken to detect all bacteria and fungi was 1-2 days earlier than that detected by the conventional blood culture method. Recovery of Gramnegative bacilli was comparable with both the methods. The commercially available Isolator system (Wampole/Dupont) is definitely better than the method used in this study in recovering blood culture isolates.
There is still no single blood culture system capable of offering optimal recovery of aerobic and facultatively anaerobic bacteria, anaerobic bacteria, and yeasts. Thus, the use of at least two blood culture bottles or systems is advocated. ${ }^{[0]}$ The lysis centrifugation method minimizes contamination and has a faster recovery of bacteria and fungi, whereas the conventional blood culture method has a greater yield of microorganism.

Therefore, in resource restricted settings, where automated blood culture systems like BACTEC, BacT/ALERT, etc. are not feasible, a combination of lysis centrifugation method and conventional blood culture method with trypticase soy broth or biphasic media is advocable in order to achieve faster recovery and a better yield of microorganisms. Latter are simple methods, do not require any specialized equipments and uses chemicals and media which are readily available. The rapid identification of aetiological agents would permit the clinician to institute appropriate therapy, thereby reducing hospital stay, morbidity and mortality in patients with sepsis.

\section{REFERENCES}

1. Reimer LG, Wilson LM, Weinstein MP. Update on detection of bacteraemia and fungaemia. Clin Microbiol Rev 1997;10:444-65.

2. Hall KK, Lyman JA. Updated review of blood culture contamination. Clin Microbiol Rev 2006;19:788-802.

3. Borriello SP, Murray RP, Funke G. In: Topley and Wilson's Microbiology and Microbial Infections: Bacteriology. 10 ${ }^{\text {th }}$ ed. Vol. 1 and 2. London: Hodder Arnold; 2005. p. 509-54;811.

4. Forbes BA, Sahm DF, Weissfeld AS. In: Bailey and Scott's Diagnostic Microbiology. $12^{\text {th }}$ ed. Missouri: Mosby Elsevier; 2007. p. 871-83.

5. Henry NK, McLimans CA, Wright AJ, Thompson RL, Wilson WR. Washington JA $2^{\text {nd }}$. Microbiological and clinical evaluation of the isolator lysis-centrifugation blood culture tube. J Clin Microbiol 1983;17:864-9.

6. Dorn GL, Land GA, Wilson GE. Improved blood culture technique based on centrifugation. clinical evaluation. J Clin Microbiol 1979;9:391-6

7. Kelly MT, Buck GE, Fojtasek MF. Evaluation of a lysis-centrifugation and biphasic bottle blood culture system during routine use. J Clin Microbiol 1983;18:554-7.

8. Kiehn TE, Wong B, Edwards FF, Armstrong D. Comparative recovery of bacteria and yeasts from lysis-centrifugation and a conventional blood culture system. J Clin Microbiol 1983;18:300-4.

9. Henry NK, Grewell CM, Van Grevenhof PE, Ilstrup DM, Washington JA $2^{\text {nd }}$. Comparison of lysis-centrifugation with a biphasic blood culture medium for the recovery of aerobic and facultatively anaerobic bacteria. J Clin Microbiol 1984;20:413-6.

10. Mantur BG, Mangalgi SS. Evaluation of conventional castaneda and lysis centrifugation blood culture techniques for diagnosis of human brucellosis. J Clin Microbiol 2004;42:4327-8.

11. David ST, Mukundan U, Brahmadathan KN, Jacob TJ. Detecting mycobacteraemia for diagnosing tuberculosis. Indian J Med Res 2004;119:259-66.

12. Sinha K, Tendolkar U, Mathur M. Comparison of Conventional Broth Blood Culture Technique and Manual Lysis Centrifugation Technique for Detection of Fungemia. Indian J Med Microbiol 2009;27:79-80.

13. Washington WC Jr, Allen S, Janda W, Koneman E, Procop G, Shreckenberger P, et al. In: Koneman's Color Atlas and Textbook of 
Diagnostic Microbiology. $6^{\text {th }}$ ed. Philadelphia: Lippincott Williams and Wilkins; 2006. p. 100-5,1219,1443-81.

14. Cheesbrough M. District Laboratory Practice in Tropical Countries. $2^{\text {nd }}$ ed. Vol. 2. Cambridge: Cambridge University Press; 2006. p. 127.

15. Shrestha P, Das BK, Bhatta NK, Jha DK, Das B, Setia A, et al. Clinical and Bacteriological Profiles of Blood Culture Positive Sepsis in Newborns. J Nepal Paediatr Soc 2007;27:64-7.

16. Ernst DJ. Controlling blood-culture contamination rates. MLO Med Lab Obs 2004;36:14-8;quiz 20-1.

17. Raja N, Parratt D, Meyers M. Blood culture contamination in a district general hospital in the UK: a 1 - year study. Healthc Infect 2009;14:95-100

18. De A, Saraswathi K, Gogate A, Fernandes A. Bacteraemia In Hospitalised Children - A One Year Prospective Study. Indian J Med Microbiol 1995;13:72-5.

How to cite this article: Parikh HR, De AS, Baveja SM. Comparison of the Lysis Centrifugation Method with the Conventional Blood Culture Method in Cases of Sepsis in a Tertiary Care Hospital. J Lab Physicians 2012;4:89-93.

Source of Support: Nil. Conflict of Interest: None declared.

\section{Author Help: Online submission of the manuscripts}

Articles can be submitted online from http://www.journalonweb.com. For online submission, the articles should be prepared in two files (first page file and article file). Images should be submitted separately.

\section{1) First Page File:}

Prepare the title page, covering letter, acknowledgement etc. using a word processor program. All information related to your identity should be included here. Use text/rtt/doc/pdf files. Do not zip the files.

\section{2) Article File:}

The main text of the article, beginning with the Abstract to References (including tables) should be in this file. Do not include any information (such as acknowledgement, your names in page headers etc.) in this file. Use text/rtf/doc/pdf files. Do not zip the files. Limit the file size to $1024 \mathrm{~kb}$. Do not incorporate images in the file. If file size is large, graphs can be submitted separately as images, without their being incorporated in the article file. This will reduce the size of the file.

3) Images:

Submit good quality color images. Each image should be less than $\mathbf{4 0 9 6} \mathbf{~ k b ~ ( 4 ~ M B ) ~ i n ~ s i z e . ~ T h e ~ s i z e ~ o f ~ t h e ~ i m a g e ~ c a n ~ b e ~ r e d u c e d ~ b y ~ d e c r e a s i n g ~}$ the actual height and width of the images (keep up to about 6 inches and up to about $1800 \times 1200$ pixels). JPEG is the most suitable file format. The image quality should be good enough to judge the scientific value of the image. For the purpose of printing, always retain a good quality, high resolution image. This high resolution image should be sent to the editorial office at the time of sending a revised article.

4) Legends:

Legends for the figures/images should be included at the end of the article file. 